\title{
Pesquisa de marcadores precoces de Arterioesclerosis en sujetos pre-hipertensos e hipertensos.
}

Óscar Román, Ana María Dussaubat y Óscar Moreno U.

Servicio Cardiovascular. Hospital San Borja - Arriarán.

\section{Resumen:}

Introducción: Se han descrito varias alteraciones del sistema circulatorio y corazón que aparecen en forma precoz en el curso evolutivo de la hipertensión arterial, particularmente en niveles de prehipertensión y en hipertensión etapa 1.

Objetivo: Estudiar algunos marcadores de daño en pacientes hipertensos no tratados.

Métodos: Se incluyeron 2 grupos de hipertensos no tratados, uno con cifras consideradas pre-hipertensión (120-139/80-89 mmHg) en 17 casos y otro con hipertensión Etapa 1 (Consenso 7), cifras de 140160/90-100 mmHg en 47 pacientes. Se les estudiaron marcadores de daño, tales como alteraciones iniciales de fondo de ojo, ausencia de Dip nocturno en el monitoreo ambulatorio de presión arterial (PA) de 24 hrs., hipertrofia ventricular izquierda y micro albuminuria elevada en $24 \mathrm{hrs}$. Se exigió rangos estrictos en 3 registros clínicos clásicos de medición de la PA. Resultados: En ambos grupos se observó la presencia de uno o más marcadores analizados: en el $59 \%$ del grupo de pre- hipertensos y en el $68 \%$ de los pacientes hipertensos etapa 1 . Entre ambos grupos, sólo la presencia de hipertrofia ventricular fue significativamente más frecuente en los pacientes en Etapa $1(\mathrm{p}<0.05)$. Los factores de riesgo se distribuyeron con igual frecuencia en ambos grupos

Conclusión: Se concluye que en etapas precoces del curso evolutivo de la PA aparecen marcadores de alteración ateroesclerótica inicial del sistema circulatorio en más de la mitad de los pacientes, antes de la aparición de daño severo de órganos blancos. 


\section{Detection of early markers for atherosclerosis in pre-hypertensive and hypertensive subjects}

Background: Several alterations of the heart and circulatory system may appear early on during the course of hypertension, both at pre-hypertension or at stage I levels.

Aim: to determine the frequency of early markers of organ damage in hypertensive subjects, both treated and un-treated

Methods: two groups of un-treated hypertensive subjects, pre-hypertensives (blood pressure 120-139 / 80-89 $\mathrm{mmHg}, \mathrm{n}=17)$ or stage I hypertensives (140-160 / 90$100 \mathrm{mmHg}, \mathrm{n}=47$ ) were studied. Three concordant measurements of blood pressure were required for inclusion. Initial fundoscopic abnormalities, absence of nocturnal blood pressure dip on $24 \mathrm{hr}$ Holter monitoring, presence of left ventricular hypertrophy and increased $24 \mathrm{hr}$ albuminuria, were determined.

Results: Both groups exhibited one or more of the abnormalities being studied. Only left ventricular hypertrophy was more frequent in stage I hypertensives compared to pre hypertensives $(\mathrm{p}<0.05)$. Risk factors for coronary artery disease did not differ between groups.

Conclusion: More than $50 \%$ of patients with prehypertension or stage I hypertension exhibit initial markers for atherosclerotic changes in the circulatory system, before the development of severe target organ damage.

Keywords: hypertension, early organ damage.

\section{Introducción:}

Es sabido que la Ateroesclerosis, después de un largo período de incubación o subclínico, provoca complicaciones en diversos territorios arteriales sistémicos y especialmente en los órganos blanco, determinando infarto del miocardio, accidente cerebrovascular, obstrucción de grandes arterias periféricas y sus consecuencias como insuficiencia cardíaca y renal ${ }^{1,2,3,4}$. Estas complicaciones se presentan generalmente en forma brusca, sin ser precedidas por síntomas o signos prodrómicos, de modo que su reconocimiento clínico es tardío. Sin embargo, se han identificado algunas alteraciones patológicas y funcionales cuya aparición es precoz en el curso evolutivo de la enfermedad y que puede permitir una prevención y tratamiento del daño arterial sistémico. Estas alteraciones se localizan en diversos territorios arteriales y pueden ser reconocidas precozmente. Algunos autores las han denominado marcadores iniciales de arteriosclerosis ${ }^{5,6,7}$.

Es preciso reconocer que los primeros elementos indicadores de riesgo de arteriosclerosis fueron los ya clásicos factores del estudio de Framingham ${ }^{8}$, como el tabaquismo, la diabetes, obesidad e hipercolesterolemia ${ }^{9}$, de los cuales la hipertensión arterial es tal vez el más significativo ${ }^{10}$, Además se ha determinado que la magnitud del riesgo de ateroesclerosis sigue una relación directa con la elevación de las cifras de presión arterial (PA) considera- das normales a niveles inferiores a 140/90, estableciéndolas hasta 120/85 mmHg. Entre ambos valores, las cifras fueron denominadas inicialmente normales altas y luego, pre-hipertensión (Consensos Internacionales) ${ }^{13,14}$. Se ha comprobado, además un riesgo significativo cardiovascular en sujetos con esos niveles de PA7, ${ }^{15}$.

Estas observaciones han llevado a plantear la necesidad de un reconocimiento precoz, tanto de las lesiones iniciales o marcadores de daño, como de los clásicos factores epidemiológicos de riesgo cardiovascular 9,13,14. Ello hace necesario estudiar las alteraciones patológicas y fisiopatológicas cardiovasculares que han sido propuesta como marcadores iniciales de daño ateroesclerótico sistémico ${ }^{7}$. Esta investigación tiene por objetivo registrar la presencia de marcadores precoces de compromiso ateroesclerótico y de factores de riesgo clásicos (FR) en los pacientes referidos a la Unidad de Hipertensión Arterial cuyas cifras de PA estuvieran en los rangos de pre-hipertensión y de hipertensión en etapa 1 según el Consenso $7^{\circ}$.

\section{Material y Método:}

Entre los meses de Enero y Noviembre de 2009, los pacientes referidos desde los consultorios periféricos de atención primaria a la Unidad de Hipertensión Arterial del Hospital San Borja Arriarán, por presentar presiones arteriales variables, a veces sobre y otras por debajo de 
los límites clásicos de normo tensión o por sospecha definida hipertensión. Fueron sometidos a un tamizaje previo consistente en 3 registros ambulatorios de la PA cada 15 días, entre las 07 y 09 hrs, en posición sentada y con los resguardos operativos clásicos. Los registros fueron realizados en ausencia de terapia farmacológica, y si en los hipertensos reconocidos, ésta había sido instituida previamente, fue suspendida por un mes, observando que las elevaciones de PA registradas no aumentaran en forma significativa. Conjuntamente se registraron 7 de los factores de riesgo clásicos (tabaquismo, diabetes, obesidad, alcohol, hiperlipidemia, gota y sedentarismo).

Los pacientes fueron divididos en dos grupos de acuerdo a los rangos de PA sistólica y diastólica definidos como pre-hipertensión (130-139/80-89 mmHg), e hipertensión etapa 1 (140-160/90-100 mmHg), de acuerdo a Consenso $7^{\circ}$. Un total de 17 pacientes $(5 \mathrm{H}$ y $12 \mathrm{M})$ fueron reclutados en el primer grupo y $47(17 \mathrm{H}$ y $30 \mathrm{M})$ en el segundo. Las edades promedio fueron $50 \pm 7,5$ y $54 \pm 8.0$, respectivamente. En todos los pacientes, además de la historia clínica, se practicaron exámenes en busca de 4 reconocidos marcadores precoces 7, a saber: alteraciones iniciales del Fondo de Ojo, ausencia de Dip nocturno en el Monitoreo de PA de 24 horas (MAPA), hipertrofia ventricular izquierda (HVI) al Ecocardiograma y Microalbuminuria de 24 horas sobre los valores normales (hasta $80 \mathrm{mg} / 24 \mathrm{hrs}$ ).

En el fondo de ojo se consideraron alteraciones iniciales el aumento de brillo arterial, espasmo y/o cruces veno-arteriales escasas. En el ecocardiograma se valoró solamente la presencia de hipertrofia septal aislada o concéntrica y no el crecimiento de la Aurícula izquierda o trastornos de la relajación diastólica. En el MAPA se valoró sólo la ausencia de "Dip" o caída nocturna de la PA.

Se excluyeron los pacientes que hubiesen presentado previamente daño significativo de órganos blancos, como IAM, AVE, Insuficiencias renal o cardíaca y compromiso atereosclerótico de extremidades inferiores o de carótidas.

\section{Resultados:}

En la Tabla 1 se presenta la frecuencia de aparición de los marcadores precoces en los dos grupos de pacientes y en la Tabla 2, la frecuencia de los factores de riesgo.

Se observa que un 59\% de los Pre-hipertensos (10 pac.) mostraron marcadores precoces y un $68 \%$ (34 pac) de los hipertensos en Etapa 1.

Se registraron 14 marcadores en 10 pacientes pre-hipertensos, uno sólo en 6 casos y dos en los 4 restantes. La distribución del tipo de marcador fue muy similar, por cuanto cada uno apareció 3 veces, con excepción de la microalbuminuria elevada que lo hizo 5 veces (Tabla1).

\begin{tabular}{|c|c|c|c|c|c|}
\hline & \multicolumn{2}{|c|}{$\begin{array}{l}\text { Pre-Hipertensos } \\
\qquad \mathrm{N}=17\end{array}$} & \multicolumn{3}{|c|}{$\begin{array}{l}\text { Hipertensos Etapa I } \\
\qquad \mathrm{N}=47\end{array}$} \\
\hline & $\mathbf{N}$ & $\%$ & $\mathbf{N}$ & $\%$ & $\mathbf{P}$ \\
\hline Fondo de Ojo alterado & 3 & 17 & 13 & 27,5 & NS \\
\hline Dip nocturno ausente & 3 & 17 & 11 & 23 & NS \\
\hline Hipertrofia VI & 3 & 17 & 19 & 40 & 0.02 \\
\hline Microalbuminuria & 5 & 29 & 13 & 27.5 & NS \\
\hline TOTAL & 14 & & 56 & & \\
\hline
\end{tabular}

En los hipertensos en Etapa 1, se registraron 56 marcadores en 32 pacientes, cuya distribución fue también muy similar, aunque predominó la presencia de hipertrofia ventricular izquierda (Tabla 1). Individualmente, 25 pac. Presentaron menos de 2 marcadores, y 7 pac., 3 o 4 marcadores simultáneamente.

Al comparar la frecuencia con que se presentaron los marcadores en ambos grupos de hipertensos, se observa que los hipertensos Etapa 1 tienen una discreta mayor frecuencia de aparición de los marcadores analizados, pero la diferencia no fue significativa con excepción de la hipertrofia ventricular izquierda $(\mathrm{p}<0.02)$.

Respecto a los factores de riesgo (FR), no hubo diferencia entre las frecuencias de aparición de ellos en ambos grupos de pacientes (Tabla2). Individualmente la mayoría de los pacientes de ambos grupos no presentaban más de 2FR, y sólo 3 de cada grupo registraron 3 y 4 FR simultáneamente.

\begin{tabular}{|c|c|c|c|c|c|}
\hline & \multicolumn{2}{|c|}{$\begin{array}{l}\text { Pre-Hipertensos } \\
\qquad N=17\end{array}$} & \multicolumn{2}{|c|}{$\begin{array}{l}\text { Hipertensos Etapa I } \\
\qquad N=47\end{array}$} & \multirow[t]{2}{*}{$p$} \\
\hline & $\mathbf{N}$ & $\%$ & $\mathbf{N}$ & $\%$ & \\
\hline Tabaquismo & 4 & 23.5 & 12 & 25.5 & NS \\
\hline Dislipidemia & 4 & 23.5 & 15 & 32 & NS \\
\hline Diabetes & 0 & - & 4 & 8 & -- \\
\hline Obesidad & 2 & 12 & 3 & 6 & NS \\
\hline Alcohol & 0 & - & 4 & 8 & - \\
\hline Sedentarismo & 10 & 59 & 33 & 70 & NS \\
\hline TOTAL & 20 & & 71 & & \\
\hline
\end{tabular}

\section{Discusión:}

En la búsqueda de marcadores precoces de Ateroesclerosis en pre-hipertensos hemos observado su presencia en poco más de la mitad (59\%) de los casos estudiados. Ello está en concordancia con la observación de otros autores, 
que han comunicado estas alteraciones en pacientes con pequeñas elevaciones de la $\mathrm{PA}^{7,15}$. Similar hallazgo se registró en pacientes definidamente hipertensos, en Etapa 1 (Consenso $7^{\circ}$ ), pero su frecuencia fue mayor $(68 \%)$. Es posible que esta diferencia tenga relación con la mayor elevación de la PA en el segundo grupo, o bien sea debido a la influencia del tiempo de evolución del estado hipertensivo. Desafortunadamente, no tenemos información de la fecha de comienzo de la HTA, pero sí podemos presumir que este factor no está relacionado con la edad de los pacientes, que en promedio y dispersión fue muy similar en ambos grupos (50 y 54 años, respectivamente).

Dado que el reclutamiento de los pacientes fue realizado al azar y simultáneamente en ambos grupos, se observó que el número de hipertensos fue mayor que el de los prehipertensos. Por tanto, pudiera pensarse que epidemiológicamente los pre-hipertensos son menos frecuentes. Sin embargo, los datos de la literatura muestran un porcentaje mayor de pre-hipertensos (en promedio 30 a 35\%) que hipertensos iniciales $(20 \%)^{16,17}$.

La comprobación de alteraciones precoces de daño ateroesclerótico pudiera correlacionarse con la mayor o menor frecuencia de presentación de los factores de riesgo reconocidos. Sin embargo, la incidencia de FR no fue significativamente mayor en pre-hipertensos que en hipertensos, puesto que individualmente se registró un número similar de FR ( 2 a 4 FR en los pacientes de cada grupo) y un mismo porcentaje de ausencia de ellos (15\%). En la literatura tampoco se han observado diferencias significativas en la incidencia de FR en pre-hipertensos en relación a hipertensos ya establecidos ${ }^{7,15}$.

Es preciso reconocer que hemos estudiado sólo un número reducido de marcadores (4 de ellos), en circunstancias que se han descrito otros de aparente similar importancia: aumento de la presión de pulso, aumento del tamaño y presión de aurícula izquierda, aumento del espesor de la pared carotídea, reducción de la velocidad de filtración glomerular y disfunción endotelial ${ }^{6,7}$. Esta limitación se justifica porque hemos privilegiado los marcadores técnicamente frecuentes de reconocer en la práctica clínica habitual.

De acuerdo a otras investigaciones, la presencia de marcadores precoces de daño arterial en pre-hipertensos se correlaciona con un mayor riesgo de complicaciones en comparación a sujetos normotensos ${ }^{15,18,19}$. Esta comprobación ha llevado a postular que los pacientes pre-hipertensos debieran ser considerados definitivamente hipertensos, habiéndose propuesto esta etapa como inicial y denominada Etapa 1 por el Hipertensión Writing Group ${ }^{7}$. Debemos reconocer también que nuestro criterio para definir los rangos de pre-hipertensión y aún de hipertensión Etapa 1 han sido eminentemente clínicos, con promedios de registro en la consulta hospitalaria. Aunque contamos simultáneamente con monitoreo Ambulatorio de 24 hrs (MAPA), no lo utilizamos para este propósito porque éste demuestra frecuentemente cifras promedio diurnas ligeramente inferiores a las registradas en la consulta hospitalaria clásica ${ }^{20}$.

Otro elemento a considerar en el establecimiento de las etapas de la Hipertensión es la gran variabilidad de las cifras de la PA y por tanto, la escasa precisión para delimitar sus etapas y para situarlas con permanencia en los rangos definidos por los Consensos $1^{3,14}$.

Esta problemática sustenta la sugerencia de marcadores de daño y de FR y no de las cifras de PA, habitualmente muy variables ${ }^{7,21}$.

Desde un punto de vista pronóstico, se reconoce que un gran porcentaje de pre-hipertensos que presentan uno o más FR, tienen alto riesgo de desarrollar hipertensión con cifras sobre $140 / 90 \mathrm{mmHg}{ }^{22}$. No hemos realizado un seguimiento de este grupo de pacientes, pero la presencia de un mayor número de marcadores en el grupo de hipertensos con cifras entre 140-160/90-100 mmHg, permitiría postular que ese curso evolutivo puede ser real.

Si lo anterior es válido, podría ser necesario iniciar una terapia más enérgica en los pre-hipertensos, no sólo con medidas farmacológicas y de control de los FR, sino además, con fármacos. Aunque actualmente esta conducta se preconiza en diabéticos con presiones aún normales ${ }^{12,21}$, no hay estudios comunicados que sustenten un tratamiento farmacológico precoz en los pacientes pre-hipertensos? ${ }^{7}$. Por último, como uno de los autores lo sugería hace más de una década ${ }^{23}$, la hipertensión debería ser considerada sólo como otro factor de riesgo, tal vez el más importante, de la enfermedad aateroesclerótica, de modo de establecer las etapas evolutivas del proceso patológico arterial en base a los marcadores precoces de daño y a los factores de riesgo y no tan sólo en función de las cifras de $\mathrm{PA}^{22,23}$. En suma, la aparición de marcadores precoces de daño ateroesclerótico en diversos territorios en más de la mitad de los casos de pre-hipertensos e hipertensos iniciales, hace perentorio considerar la búsqueda de ellos para definir precozmente las conductas preventivas de la enfermedad ateroesclerótica, tanto con medidas no farmacológicas como posiblemente también, farmacológicas. 


\section{Referencias:}

1.- HOLME I, ENGER SC, HELGERLAND A. Risk factors and raised atherosclerotic lesions in coronary and cerebral arteries: Statistical analysis from the Oslo Study. Atherosclerosis 1981; $1: 250-56$

2.- GIMBRONE M A, RESNICK N, TOPPER G T. Vascular endothelium, hemodynamics and atherogenesis. En "Vascular Endothelium". Schauttauer Ed. Stutgart. pags. 287-297.

3.- ROMAN O,MESAN.Arterial hypertension: end organ damage and its prevention. Intercontinental Cardiology 1996; 5:49-56.

4.- JULIUS S, JAMERSON K, MEJIA A, SCHORKA N, JONES

$\mathrm{K}$. The association of borderline hypertension with target organ changes and higher coronary risks. JAMA. 1990;264:354-58.

5.- BERLINER JA, NAVA BM, FOGELMAN AM. Atherosclerosis basic mechanism: oxidation inflammation and genetic. Circulation 1995; 91: 2488-96.

6.- BARJA S, ACEVEDO M, ANAIZ P, BARRIOS X, BAMBS C, GUZMAN B, et al. Marcadores de ateroesclerosis temprana y síndrome metabólico en niños. Rev Med Chile 2009; 137:522-30.

7.- GILES T D, BERK B C, BLACK H R, COHN J R, KOSTIS J VB, IZZO J L, et al . Expanding the definition and classification of hypertension. J Clin Hypertens 2005; 7: 505-12.

8.- CASTELLI W P, ANDERSON K. A population at risk. Prevalence of high cholesterol levels in hypertensive patients in the Framingham Study. Am J Med 1986;80: 23-32.

9.- POOLING PROJECT RESEARCH GROUP. Relationship of blood pressure, serum cholesterol, smoking habit, relative weight and ECG abnormalities of major coronary events. Final report of the Pooling Project Research Group. J Chronic Dis 1975;31.201-306.

10.- KANNEL W B. Importance of hypertension as a major risk factor in cardiovascular disease. In Hypertension, 1977 Mc Graw Hill, pags 88-91.
11.- MAC MAHONS S, PETO R, CUTLER J. Blood pressure, stroke and coronary heart disease, Part 1 . Prolonged differences in blood pressure prospective observational studies corrected for the regression dilution bias. Lancet 1990; 335: 765-73.

12.- HANSSON L, ZANCHETTI A, CARRUTHERS S, DAHLOF B, ELMFELD T, JULIUS S, et al. Effect of intensive blood pressure lowering and low dose aspirin in patients with hypertension: principal results of the Hypertension optimal Treatment (HOT) randomized trial. Lancet 1998; 351;1755-62.

13.- JOINT NATIONAL COMMITTEE ON PREVENTION, DETECTION, EVALUATION AND TREATMENT OF HIGH BLOOD PRESSURE. The sixth report. Arch Intern Med 1997; 157:2413-46.

14.- CHOBANIAN AV, BAKRIS GL, BLACK HR, CUSHMAN WC, GREEN LA, IZZO JL JR, et al. Seventh report of the Joint National Committee on Prevention, Evaluation and Treatment of High Blood Pressure. Hypertension 2003; 42:1206-52.

15.- VASAN RS, LARSON MG, LEIP EP, EVANS JC, O'DONNELL CJ, KANNEL WB, et al. Impact of high normal blood pressure on the risk of cardiovascular disease. $\mathrm{N}$ Engl $\mathrm{J}$ Med 2001; 345:1291-97.

16.- AEKPLAKORN W, ABBOTT-KLAFTER J, KHONPUTSA P, TATSANAVIVAT P, CHONGSUVIVATWONG V, CHARIYALERTSAK S, et al. Prevalence and management of pre-hypertension and hypertension by geographic regions of Thailand. J. Hypertens 2008; 26:191-98.

17.- GODWIN M, PYKE A, KIRLY A, JEWER C, MURPHY L. Pre-hypertension and hypertension in a primary care practice. Can Fam Physician 2008; 54:1418-23.

18.- PITTARAS A, MYERS J, NARAYAN L, MANOLIS R. Exercise capacity is inversely associated with mortality in pre-hypertension. Am Heart Assoc. Meeting 2008 New Orleans.

19.- MAINOUS AG, EVERET CJJ, LISZKA H. Prehypertension and mortality in a national representative cohort. Am J Cardiol 
2001; 94: 1496-1500.

20.- PRAT H, VALDES G, ROMAN O, ZARATE LH. Actualización de las recomendaciones sobre el uso de la monitorización ambulatoria de presión arterial Documento de Consenso de la Sociedad Chilena de Hipertensión. 2009.

21.- ROMAN O, BADILLA M, DUSSAUBAT A M. La Hipertensión arterial ¿solo un biomarcador de la enfermedad ateroesclerótica sistémica? Rev. Med Chile 2010;
138:346-51.

22.- VASAN R S, BEISER A, SESHADRI S, Residual life time risk for developing hypertension in middle-aged women an men: The Framingham Herat Study. JAMA 2001; 287: 1001-1010.

23.- ROMAN O, La enfermedad cardiovascular ¿una nueva entidad? Relación de la hipertensión arterial con ella. Bol Soc Ch Hipertens 1998; 7:3-5. 